Algebraic $\& \mathcal{G}$ Geometric Topology

Volume 2 (2002) 371-380

ATG

Published: 21 May 2002

\title{
Intrinsic knotting and linking of complete graphs
}

\author{
ERICA FLAPAN
}

\begin{abstract}
We show that for every $m \in \mathbb{N}$, there exists an $n \in \mathbb{N}$ such that every embedding of the complete graph $K_{n}$ in $\mathbb{R}^{3}$ contains a link of two components whose linking number is at least $m$. Furthermore, there exists an $r \in \mathbb{N}$ such that every embedding of $K_{r}$ in $\mathbb{R}^{3}$ contains a knot $Q$ with $\left|a_{2}(Q)\right| \geq m$, where $a_{2}(Q)$ denotes the second coefficient of the Conway polynomial of $Q$.
\end{abstract}

AMS Classification 57M25; 05C10

Keywords Embedded graphs, intrinsic knotting, intrinsic linking

\section{Introduction}

The study of intrinsic knotting and linking began with the work of Conway and Gordon $[\mathrm{CG}]$, who showed that every embedding in $\mathbb{R}^{3}$ of the complete graph on six vertices, $K_{6}$, contains a non-trivial link of two components, and every embedding of $K_{7}$ in $\mathbb{R}^{3}$ contains a non-trivial knot. Since the existence of such a link or knot is intrinsic to the graph, and does not depend on the particular embedding of the graph in $\mathbb{R}^{3}$, we say that $K_{6}$ is intrinsically linked and $K_{7}$ is intrinsically knotted. We state Conway and Gordon's Theorem more precisely with the following notation. Let $L_{1} \cup L_{2}$ be an oriented link, and let $l k\left(L_{1}, L_{2}\right)$ denote the linking number of $L_{1}$ and $L_{2}$; let $Q$ be a knot, and let $a_{2}(Q)$ denote the second coefficient of the Conway polynomial of $Q$. Conway and Gordon proved that every embedding of $K_{6}$ contains a link $L_{1} \cup L_{2}$ which has $l k\left(L_{1}, L_{2}\right) \equiv 1(\bmod 2)$, and every embedding of $K_{7}$ contains a knot $Q$ which has $a_{2}(Q) \equiv 1(\bmod 2)$. Furthermore, they illustrated an embedding of $K_{6}$ such that the only non-trivial link $L_{1} \cup L_{2}$ contained in $K_{6}$ is the Hopf link (which has $\left|l k\left(L_{1}, L_{2}\right)\right|=1$ ); and they illustrated an embedding of $K_{7}$ such that the only non-trivial knot $Q$ contained in $K_{7}$ is the trefoil knot (which has $\left.\left|a_{2}(Q)\right|=1\right)$. In this sense $K_{6}$ exhibits the simplest type of intrinsic linking and $K_{7}$ exhibits the simplest type of intrinsic knotting.

In this paper, we will show that for larger values of $n$, the complete graph $K_{n}$ can exhibit a more complex type of intrinsic linking or knotting. In particular, 
there exists an $n$ such that every embedding of $K_{n}$ contains a non-trivial 2component link which is not the Hopf link, and there exists an $n$ such that every embedding of $K_{n}$ contains a non-trivial knot which is not the trefoil knot. In [FNP] we considered links of more than two components, and showed that every embedding of $K_{10}$ in $\mathbb{R}^{3}$ contains a 3-component link $L_{0} \cup L_{1} \cup L_{2}$ such that both $l k\left(L_{0}, L_{1}\right) \equiv 1(\bmod 2)$ and $l k\left(L_{0}, L_{2}\right) \equiv 1(\bmod 2)$; furthermore, $n=10$ is the smallest number such that $K_{n}$ has this property. Here we shall generalize Conway and Gordon's Theorem by considering the linking number of 2-component links in $\mathbb{Z}$ rather than in $\mathbb{Z}_{2}$, and by considering the second coefficient of the Conway polynomial in $\mathbb{Z}$ rather than in $\mathbb{Z}_{2}$.

We begin by proving that every embedding of $K_{10}$ contains a non-trivial link of two components other than the Hopf link. In particular we prove the following.

Theorem 1 Every embedding of $K_{10}$ in $\mathbb{R}^{3}$ contains a 2-component link $L=$ $L_{1} \cup J_{1}$ such that for some orientation of $L$ we have $l k\left(L_{1}, J_{1}\right) \geq 2$.

Theorem 2 will show that the complexity of the intrinsic linking of $K_{n}$ (as measured by the linking number) can be made as large as we wish by making $n$ sufficiently large.

Theorem 2 Let $p \in \mathbb{N}$ be given, and let $n=p(15 p-9)$. Then every embedding of $K_{n}$ in $\mathbb{R}^{3}$ contains a 2-component link $L=L_{p} \cup J_{p}$ such that for some orientation of $L$ we have $l k\left(L_{p}, J_{p}\right) \geq p$.

It is natural to ask whether the complexity of the intrinsic knotting of $K_{n}$ can also be made as large as we wish by choosing $n$ sufficiently large. While the linking number seems like the natural measure of the complexity of a 2component link, there are various ways to measure the complexity of a knot. The second coefficient of the Conway polynomial $a_{2}(Q)$ of an oriented knot $Q$ is a convenient invariant to use because it relates knotting and linking. In particular, Kauffman [Ka] has shown that it satisfies the following equation

$$
a_{2}\left(K_{+}\right)=a_{2}\left(K_{-}\right)+l k\left(L_{1}, L_{2}\right)
$$

where $K_{+}$and $K_{-}$are identical oriented knots outside of the crossing illustrated in Figure 1, and the oriented link $L_{1} \cup L_{2}$ is obtained by smoothing this crossing as illustrated.

Due to the utility of equation (1), the second coefficient of the Conway polynomial has been used to prove various theorems about knots contained in spatial graphs (see for example [CG], [Fo], [TY], [Sh]). Using $a_{2}(Q)$ as a measure of knot complexity we prove the following theorem. 

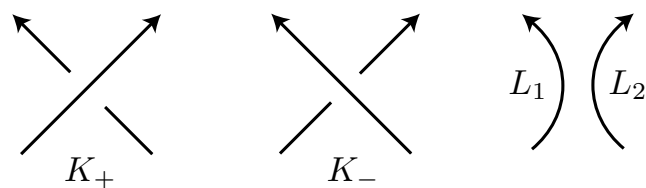

Figure 1: The skein moves

Theorem 3 Let $m \in \mathbb{N}$ be given, let $p$ be an integer such that $p \geq 4 \sqrt{m}$, and let $n=p(15 p-9)$. Then every embedding of $K_{2 n}$ in $\mathbb{R}^{3}$ contains a knot $Q$ with $\left|a_{2}(Q)\right| \geq m$.

Observe that by picking $p$ to be the smallest integer such that $p \geq 4 \sqrt{m}$, we have $p \leq 4 \sqrt{m}+1$ and hence $2 n \leq 720 m-60$. In particular, this means that $2 n$ only grows linearly with $m$.

The question of finding the minimum number of vertices necessary to guarantee a certain type of intrinsic linking or knotting remains open. Since $K_{5}$ does not have enough vertices to contain a link, $n=6$ is the smallest number of vertices necessary such that every embedding of $K_{n}$ contains a link with $L_{1} \cup L_{2}$ with $\left|l k\left(L_{1}, L_{2}\right)\right| \geq 1$. Theorem 1 shows every embedding of $K_{10}$ in $\mathbb{R}^{3}$ contains a link with $L_{1} \cup L_{2}$ with $\left|l k\left(L_{1}, L_{2}\right)\right| \geq 2$. However, it is not known whether 10 is the smallest such $n$. In general, it is an open question to find a function $\varphi: \mathbb{N} \rightarrow \mathbb{N}$ such that for every $p \in \mathbb{N}, \varphi(p)$ is the minimum number of vertices necessary such that every embedding of $K_{\varphi(p)}$ contains a 2-component link $L_{1} \cup L_{2}$ with $\left|l k\left(L_{1}, L_{2}\right)\right| \geq p$. With respect to intrinsic knotting, the embedding of $K_{6}$ given by Conway and Gordon contains no non-trivial knot, thus $n=7$ is the minimum number of vertices necessary such that every embedding of $K_{n}$ contains a knot $Q$ with $\left|a_{2}(Q)\right| \geq 1$. It follows from Theorem 3 that every embedding of $K_{972}$ in $\mathbb{R}^{3}$ contains a non-trivial knot other than the trefoil knot. However it is not known what the smallest $n$ is such that every embedding of $K_{n}$ in $\mathbb{R}^{3}$ contains a knot other than the trefoil knot. Furthermore, it is an open question to find a function $\psi: \mathbb{N} \rightarrow \mathbb{N}$ such that for every $m \in \mathbb{N}, \psi(m)$ is the minimum number of vertices necessary such that every embedding of $K_{\psi(m)}$ contains a knot $Q$ with $\left|a_{2}(Q)\right| \geq m$.

\section{Intrinsic Linking}

The following lemma allows us to go from a 3-component link (with a sufficient number of vertices) to a 2-component link whose linking number is at least the sum of the linking numbers of two pairs of components of the 3-component link. 
Lemma 1 Let $L \cup Z \cup W$ be a 3-component link contained in some embedding of $K_{n}$ in $\mathbb{R}^{3}$. Suppose that $l k(L, Z)=p_{1}>0$ and $l k(L, W)=p_{2}>0$ for some orientation of $L \cup Z \cup W$. Suppose that $Z$ and $W$ each contain at least $q$ vertices and $q>p_{1}+p_{2}$. Then $K_{n}$ contains a simple closed curve $J$ with at least $2 q$ vertices which is disjoint from $L$ such that, for some orientation of $L \cup J$, we have $l k(L, J) \geq p_{1}+p_{2}$.

Proof On $Z$ we select $q$ vertices and label them consecutively by $v_{1}, \ldots, v_{q}$ in such a way that $Z$ is oriented in the direction of increasing order of the $v_{j}$. On $W$ we select $q$ vertices and label them consecutively by $w_{1}, \ldots, w_{q}$ in such a way that $W$ is oriented in the direction of decreasing order of the $w_{j}$. For each $j=1, \ldots, q$, let $A_{j}$ denote the simple closed curve $\overline{v_{j} w_{j} w_{j+1} v_{j+1} v_{j}}$, where the subscripts are taken $\bmod q$. We orient each $A_{j}$ so that going from $v_{j}$ to $w_{j}$ along the edge $\overline{v_{j} w_{j}}$ is the positive direction.

Now in the homology group $H_{1}\left(\mathbb{R}^{3}-L ; \mathbb{Z}\right)$ we have the equation

$$
[Z]+[W]+\left[A_{1}\right]+\ldots+\left[A_{q}\right]=0 .
$$

Thus

$$
p_{1}+p_{2}=[Z]+[W]=-\left[A_{1}\right]-\ldots-\left[A_{q}\right] .
$$

Since $\left[A_{j}\right]$ is an integer for each $j$ and $q>p_{1}+p_{2}$, there is some $j$ such that $\left[A_{j}\right] \geq 0$. Without loss of generality $\left[A_{q}\right] \geq 0$. Hence in $H_{1}\left(\mathbb{R}^{3}-L ; \mathbb{Z}\right)$ we have the inequality

$$
-\left[A_{1}\right]-\ldots-\left[A_{q-1}\right] \geq p_{1}+p_{2} .
$$

Now let $J$ denote the simple closed curve obtained from $A_{1} \cup \ldots \cup A_{q-1}$ by omitting the edges $\overline{v_{j} w_{j}}$ for $j=2, \ldots, q-1$. We orient $J$ so that going from $w_{1}$ to $v_{1}$ along the edge $\overline{w_{1} v_{1}}$ is the positive direction. Then in $H_{1}\left(\mathbb{R}^{3}-L ; \mathbb{Z}\right)$ we have

$$
[J]=-\left[A_{1}\right]-\ldots-\left[A_{q-1}\right] .
$$

Hence $l k(L, J) \geq p_{1}+p_{2}$ and $J$ has at least $2 q$ vertices.

We shall prove Theorem 1 by using Lemma 1 together with [FNP].

Theorem 1 Every embedding of $K_{10}$ in $\mathbb{R}^{3}$ contains a 2-component link $L \cup J$ such that, for some orientation, we have $l k(L, J) \geq 2$.

Proof Let $K_{10}$ be embedded in $\mathbb{R}^{3}$. It follows from [FNP] that $K_{10}$ contains a 3-component link $L \cup Z \cup W$ such that $l k(L, Z) \equiv 1(\bmod 2)$ and $l k(L, W) \equiv 1$ $(\bmod 2)$. We orient the link $L \cup Z \cup W$ so that $l k(L, Z)=p_{1} \geq 1$ and 
$l k(L, W)=p_{2} \geq 1$. If either $p_{i} \geq 3$ we are done. Otherwise, $p_{1}=p_{2}=1$. Clearly $Z$ and $W$ each have at least 3 vertices. Now we apply Lemma 1 to obtain a simple closed curve $J$ such that $l k(L, J) \geq 2$.

Lemma 2 will allow us to go from a pair of disjoint 2-component links (each with a sufficient number of vertices) to a 3 -component link, while bounding the linking numbers of the 3-component link below. The proof of Lemma 2 is similar in flavor to the proof of Lemma 1, though the details differ.

Lemma 2 Let $X_{1} \cup Y_{1} \cup X_{2} \cup Y_{2}$ be a 4-component link contained in some embedding of $K_{n}$ in $\mathbb{R}^{3}$. Suppose that for some orientation of $X_{1} \cup Y_{1} \cup X_{2} \cup Y_{2}$ we have $l k\left(X_{1}, Y_{1}\right) \geq 1$ and $l k\left(X_{2}, Y_{2}\right)=p \geq 1$. Also suppose that $X_{1}, Y_{1}$, $X_{2}, Y_{2}$ each contain at least $q$ vertices and $q>p$. Then $K_{n}$ contains disjoint simple closed curves $L, Z$ and $W$, each with at least $q$ vertices, such that $l k(L, Z) \geq 1$ and $l k(L, W) \geq p$ for some orientation of $L \cup Z \cup W$.

Proof If $l k\left(X_{2}, Y_{1}\right)$ is non-zero, then let $L=X_{2}, Z=Y_{1}$, and $W=Y_{2}$. Now if we orient $L \cup Z \cup W$ appropriately we get a link with $l k(L, Z) \geq 1$ and $l k(L, W) \geq p$. If $l k\left(Y_{2}, X_{1}\right)$ is non-zero, let $L=Y_{2}, Z=X_{1}$, and $W=X_{2}$. Then $L \cup Z \cup W$ is the desired link. So from now on, we shall assume that both $l k\left(Y_{1}, X_{2}\right)=0$ and $l k\left(X_{1}, Y_{2}\right)=0$.

We choose $q$ vertices on each of $X_{1}$ and $X_{2}$ and label the vertices of $X_{1}$ and $X_{2}$ as we did the vertices of $Z$ and $W$ in the proof of Lemma 1 . We also define the oriented simple closed curves $A_{j}$ as we did in the proof of Lemma 1. Now for both $i=1$ and $i=2$, in the first homology groups $H_{1}\left(\mathbb{R}^{3}-Y_{i} ; \mathbb{Z}\right)$ we have the equation

$$
\left[X_{1}\right]+\left[X_{2}\right]+\left[A_{1}\right]+\ldots+\left[A_{q}\right]=0 .
$$

Now by our assumptions that $l k\left(Y_{1}, X_{2}\right)=0$ and $l k\left(X_{1}, Y_{2}\right)=0$, we have $\left[X_{2}\right]=0$ in $H_{1}\left(\mathbb{R}^{3}-Y_{1} ; \mathbb{Z}\right)$ and we have $\left[X_{1}\right]=0$ in $H_{1}\left(\mathbb{R}^{3}-Y_{2} ; \mathbb{Z}\right)$. Thus in $H_{1}\left(\mathbb{R}^{3}-Y_{2} ; \mathbb{Z}\right)$ we have

$$
0<p=\left[X_{2}\right]=-\left[A_{1}\right]-\ldots-\left[A_{q}\right] .
$$

Since $q>p$, without loss of generality $\left[A_{q}\right] \geq 0$ in $H_{1}\left(\mathbb{R}^{3}-Y_{2} ; \mathbb{Z}\right)$. Hence

$$
-\left[A_{1}\right]-\ldots-\left[A_{q-1}\right] \geq p
$$

in $H_{1}\left(\mathbb{R}^{3}-Y_{2} ; \mathbb{Z}\right)$.

In $H_{1}\left(\mathbb{R}^{3}-Y_{1} ; \mathbb{Z}\right)$ we have

$$
\left[X_{1}\right]=-\left[A_{1}\right]-\ldots-\left[A_{q}\right] .
$$


First we suppose that $\left[A_{q}\right]=0$ in $H_{1}\left(\mathbb{R}^{3}-Y_{1} ; \mathbb{Z}\right)$. So $-\left[A_{1}\right]-\ldots-\left[A_{q-1}\right]=$ $\left[X_{1}\right] \geq 1$ in $H_{1}\left(\mathbb{R}^{3}-Y_{1} ; \mathbb{Z}\right)$. In this case, we let $L$ denote the simple closed curve obtained from $A_{1} \cup \ldots \cup A_{q-1}$ by omitting the edges $\overline{v_{j} w_{j}}$ for $j=2, \ldots, q-1$. We orient $L$ so that going from $w_{1}$ to $v_{1}$ along the edge $\overline{w_{1} v_{1}}$ is the positive direction. Then $L$ has at least $2 q$ vertices and $l k\left(L, Y_{1}\right)=l k\left(X_{1}, Y_{1}\right) \geq 1$ and $\left|l k\left(L, Y_{2}\right)\right| \geq p$. We are done by letting $Z=Y_{1}$ and $W=Y_{2}$.

Now suppose that $\left[A_{q}\right] \neq 0$ in $H_{1}\left(\mathbb{R}^{3}-Y_{1} ; \mathbb{Z}\right)$. Let $L$ denote the simple closed curve obtained from $X_{2} \cup A_{q}$ by omitting the edge $\overline{w_{q} w_{1}}$. We orient $L$ so that going from $v_{1}$ to $w_{1}$ along the edge $\overline{v_{1} w_{1}}$ is the positive direction. Then $L$ has at least $q+2$ vertices. Also since $l k\left(X_{2}, Y_{1}\right)=0$ we have $\left|l k\left(L, Y_{1}\right)\right|=\left|l k\left(A_{q}, Y_{1}\right)\right| \geq 1$; Now by changing the orientation on $Y_{1}$, if necessary, $l k\left(L, Y_{1}\right) \geq 1$. Since $\left[A_{q}\right] \geq 0$ in $H_{1}\left(\mathbb{R}^{3}-Y_{2} ; \mathbb{Z}\right)$ we have $l k\left(L, Y_{2}\right) \geq l k\left(X_{2}, Y_{2}\right)=p$. So we are done by letting $Z=Y_{1}$ and $W=Y_{2}$.

Now we will use Lemmas 1 and 2, together with an inductive argument, to prove Theorem 2 .

Theorem 2 Let $p \in \mathbb{N}$ be given, and let $n=p(15 p-9)$. Then every embedding of $K_{n}$ in $\mathbb{R}^{3}$ contains a 2 -component link $L_{p} \cup J_{p}$ such that for some orientation of $L_{p} \cup J_{p}$, we have $l k\left(L_{p}, J_{p}\right) \geq p$.

Proof Suppose that for every oriented link $L_{p} \cup J_{p}$ contained in $K_{n}$ we have $l k\left(L_{p}, J_{p}\right)<p$.

Let $G_{1}, \ldots, G_{p}$ be $p$ disjoint copies of $K_{15 p-9}$ which are contained in $K_{n}$. For each $i=1, \ldots, p$, let $H_{i}$ be a subgraph of $G_{i}$ containing all $15 p-9$ vertices of $G_{i}$ such that, as a topological space, $H_{i}$ is homeomorphic to $K_{6}$, yet between every pair of vertices which have valence 5 in $H_{i}$ there is a path in $H_{i}$ containing $p-1$ vertices which are each of valence 2 in $H_{i}$. Since $K_{6}$ contains 15 edges and $15(p-1)+6=15 p-9$, there is such a subgraph $H_{i}$ in $K_{15 p-9}$. Now by [CG], each $H_{i}$ contains a link $X_{i} \cup Y_{i}$ such that with some orientation we have $l k\left(X_{i}, Y_{i}\right) \geq 1$ and $X_{i}$ and $Y_{i}$ each contain 3 vertices with valence 5 and $3(p-1)$ vertices with valence 2 in $H_{i}$. Thus $X_{i}$ and $Y_{i}$ each contain a total of $3 p$ vertices.

We will prove by induction that for every $m=1, \ldots, p$, the $K_{m(15 p-9)}$, which has all of its vertices in $\bigcup_{i=1}^{m} G_{i}$, contains a link $L_{m} \cup J_{m}$ such that, with some orientation, $l k\left(L_{m}, J_{m}\right)=p_{m} \geq m$ and $L_{m}$ and $J_{m}$ each have at least $3 p$ vertices. We saw above that this is true for $m=1$. Assume that it's true for $m$. Thus $l k\left(L_{m}, J_{m}\right)=p_{m}$, and by our initial assumption $p_{m}<p$. 
Also $G_{m+1}$ is disjoint from $K_{m(15 p-9)}$ and contains a pair of simple closed curves $X_{m+1}$ and $Y_{m+1}$ each with $3 p$ vertices such that $l k\left(X_{m+1}, Y_{m+1}\right) \geq 1$. Thus the disjoint simple closed curves $L_{m}, J_{m}, X_{m+1}$, and $Y_{m+1}$ each contain at least $3 p$ vertices and $3 p>p_{m}$ since $p>p_{m}$. Thus by Lemma 2 , the $K_{(m+1)(15 p-9)}$, which has all of its vertices in $\bigcup_{i=1}^{m+1} G_{i}$, contains simple closed curves $L_{m+1}, Z_{m+1}$ and $W_{m+1}$ each with at least $3 p$ vertices such that, for some orientation of $L_{m+1} \cup Z_{m+1} \cup W_{m+1}$, we have $l k\left(L_{m+1}, Z_{m+1}\right)=q_{m} \geq 1$ and $l k\left(L_{m+1}, W_{m+1}\right)=r_{m} \geq p_{m} \geq m$. By assumption $q_{m}<p$ and $r_{m}<p$, so $q_{m}+r_{m}<3 p$. Thus we can apply Lemma 1 to obtain a simple closed curve $J_{m+1}$ which is contained in $K_{(m+1)(15 p-9)}$ such that, with some orientation, $l k\left(L_{m+1}, J_{m+1}\right) \geq q_{m}+r_{m} \geq 1+m$ and $J_{m+1}$ has at least $6 p$ vertices. Thus for all $m=1, \ldots, p$, the $K_{m(15 p-9)}$, which has all of its vertices in $\bigcup_{i=1}^{m} G_{i}$, contains a link $L_{m} \cup J_{m}$ such that, with some orientation, $l k\left(L_{m}, J_{m}\right) \geq m$ and $L_{m}$ and $J_{m}$ each have at least $3 p$ vertices. It follows that $K_{p(15 p-9)}$ contains a link $L_{p} \cup J_{p}$ such that, with some orientation, $l k\left(L_{p}, J_{p}\right) \geq p$. However this contradicts our assumption that for every oriented link $L_{p} \cup J_{p}$ contained in $K_{n}$ we have $l k\left(L_{p}, J_{p}\right)<p$. Hence we must, in fact, have a 2-component link $L_{p} \cup J_{p}$ in $K_{n}$ such that for some orientation of the link we have $l k\left(L_{p}, J_{p}\right) \geq p$.

We now observe as follows that for any given $p \in \mathbb{N}$, it is not possible to have an $n \in \mathbb{N}$, such that every embedding of $K_{n}$ contains a 2-component link with linking number equal to precisely $p$. First recall that the linking number of any oriented 2-component link is equal to one half of the sum of +1 for every positive crossing and -1 for every negative crossing. We illustrate these two types of crossings in Figure 2.
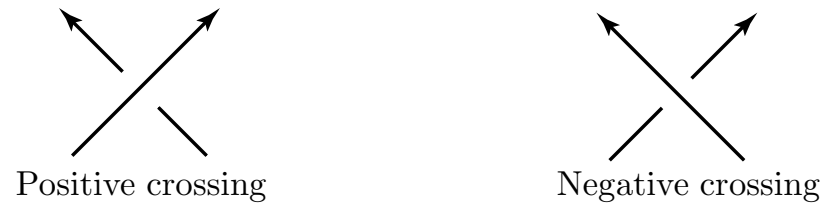

Figure 2: Positive and negative crossings

Let $n \in \mathbb{N}$ be fixed and let $K_{n}$ be embedded in $\mathbb{R}^{3}$. We shall find a (possibly different) embedding of $K_{n}$ in $\mathbb{R}^{3}$ which contains no 2-component link $L_{1} \cup L_{2}$ with $l k\left(L_{1}, L_{2}\right)=p$. Let $q=\max \left\{\left|l k\left(L_{i}, L_{j}\right)\right|\right\}$ taken over all disjoint pairs of simple closed curves $L_{i}$ and $L_{j}$, contained in $K_{n}$. Suppose there is some link $L_{1} \cup L_{2}$ in $K_{n}$ such that for some orientation, $l k\left(L_{1}, L_{2}\right)=p$. Let $e$ be an edge in $L_{1}$ and let $f$ be an edge in $L_{2}$. We create a new embedding of $K_{n}$ by adding $2 p+2 q+2$ half twists between the edges $e$ and $f$ without changing 
anything else about the embedding. We call this new embedding $K_{n}^{\prime}$ and this new pair of edges $e^{\prime}$ and $f^{\prime}$. Now for any pair of disjoint oriented simple closed curves $L_{i}$ and $L_{j}$ in $K_{n}$, there is a corresponding pair of disjoint oriented simple closed curves $L_{i}^{\prime}$ and $L_{j}^{\prime}$ in $K_{n}^{\prime}$. Furthermore, $L_{i}^{\prime}$ and $L_{j}^{\prime}$ contain $e^{\prime}$ and $f^{\prime}$ respectively if and only if $L_{i}$ and $L_{j}$ contain $e$ and $f$ respectively. Now suppose that one of $L_{i}^{\prime}$ and $L_{j}^{\prime}$ contains $e^{\prime}$ and the other contains $f^{\prime}$. Then $l k\left(L_{i}^{\prime}, L_{j}^{\prime}\right)=l k\left(L_{i}, L_{j}\right) \pm(p+q+1)$. Since $l k\left(L_{i}, L_{j}\right) \geq-q$ we know that $l k\left(L_{i}, L_{j}\right)+(p+q+1) \geq p+1>0$, so $\left|l k\left(L_{i}, L_{j}\right)+(p+q+1)\right| \geq p+1$. Since $l k\left(L_{i}, L_{j}\right) \leq q$ we know that $l k\left(L_{i}, L_{j}\right)-(p+q+1) \leq-p-1<0$, so $\left|l k\left(L_{i}, L_{j}\right)-(p+q+1)\right| \geq p+1$. Thus $\left|l k\left(L_{i}^{\prime}, L_{j}^{\prime}\right)\right|=\left|l k\left(L_{i}, L_{j}\right) \pm(p+q+1)\right| \geq$ $p+1$.

On the other hand, for any pair $L_{i}^{\prime}$ and $L_{j}^{\prime}$ which do not contain $e^{\prime}$ and $f^{\prime}$, then $l k\left(L_{i}^{\prime}, L_{j}^{\prime}\right)=l k\left(L_{i}, L_{j}\right)$. It follows that $K_{n}^{\prime}$ contains fewer links which have linking number equal to $p$ than $K_{n}$ did. If we repeat this process enough times, we will eventually have an embedding of $K_{n}$ which contains no links that have linking number equal to $p$.

\section{Intrinsic Knotting}

In order to consider intrinsic knotting of complete graphs, we shall make use of the pseudo-graph $D_{4}$ (illustrated in Figure 3) in a similar manner to that of $[\mathrm{TY}]$ and $[\mathrm{Fo}]$.

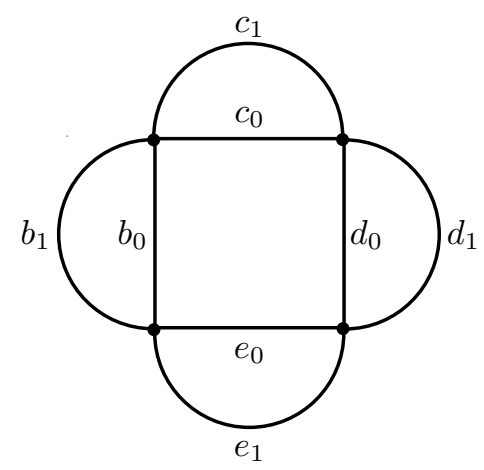

Figure 3: The pseudo-graph $G_{4}$

A Hamiltonian cycle in a graph or pseudo-graph $G$ is a simple closed curve in $G$ which contains every vertex of $G$. Each Hamiltonian cycle of $D_{4}$ has the form $b_{i} c_{j} d_{k} e_{l}$ where $i, j, k, l \in\{0,1\}$. For each such cycle we define

$$
\varepsilon\left(b_{i} c_{j} d_{k} e_{l}\right)= \begin{cases}+1 & \text { if } i+j+k+l \text { is even } \\ -1 & \text { if } i+j+k+l \text { is odd }\end{cases}
$$


Let $\Gamma$ denote the set of all Hamiltonian cycles in $D_{4}$. Recall that if $\gamma$ is a simple closed curve in $\mathbb{R}^{3}$, then $a_{2}(\gamma)$ denotes the second coefficient of the Conway polynomial of $\gamma$. For any embedding of $D_{4}$ in $\mathbb{R}^{3}$ we define

$$
\lambda=\left|\sum_{\gamma \in \Gamma} \varepsilon(\gamma) a_{2}(\gamma)\right|
$$

Let $B, C, D$, and $E$ denote the simple closed curves $b_{0} \cup b_{1}, c_{0} \cup c_{1}, d_{0} \cup d_{1}$, and $e_{0} \cup e_{1}$ respectively. It is shown in [TY] that, for a given embedding of $D_{4}$ in $\mathbb{R}^{3}$, we have the equation

$$
\lambda=|l k(E, C) l k(B, D)|
$$

Equation (2) enables us to relate knotting and linking in an embedded graph. We use this equation to prove Theorem 3 .

Theorem 3 Let $m \in \mathbb{N}$ be given, let $p$ denote an integer such that $p \geq 4 \sqrt{m}$, and let $n=p(15 p-9)$. Then every embedding of $K_{2 n}$ in $\mathbb{R}^{3}$ contains a knot $Q$ with $\left|a_{2}(Q)\right| \geq m$.

Proof Let $K_{2 n}$ be embedded in $\mathbb{R}^{3}$. Let $G_{1}$ and $G_{2}$ denote two disjoint $K_{n}$ 's which are contained in $K_{2 n}$. By Theorem 2, each $G_{i}$ contains a link $X_{i} \cup Y_{i}$ such that, for some orientation of $X_{i} \cup Y_{i}$, we have $l k\left(X_{i}, Y_{i}\right) \geq p \geq 4 \sqrt{m}$. For $i=1$ and $i=2$, let $u_{i}$ and $v_{i}$ be distinct vertices on $X_{i}$, and let $z_{i}$ and $w_{i}$ be distinct vertices on $Y_{i}$. Consider the embedded subgraph $G$ of $K_{2 n}$ consisting of $X_{1} \cup Y_{1} \cup X_{2} \cup Y_{2}$ together with the edges $\overline{v_{1} u_{2}}, \overline{w_{1} v_{2}}, \overline{z_{1} w_{2}}$, and $\overline{u_{1} z_{2}}$. Figure 4 illustrates the abstract graph $G$, with all of the other vertices omitted.

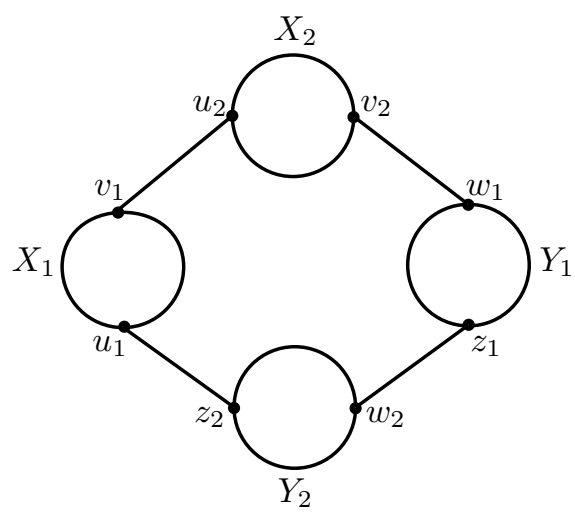

Figure 4: The graph $G$ 
From the embedded graph $G$, we obtain an embedding of the pseudo-graph $D_{4}$ by omitting all vertices of valence 2 and collapsing the edges $\overline{v_{1} u_{2}}, \overline{w_{1} v_{2}}, \overline{z_{1} w_{2}}$, and $\overline{u_{1} z_{2}}$. Now by equation (2) we have $\lambda=\left|l k\left(X_{1}, Y_{1}\right) l k\left(X_{2}, Y_{2}\right)\right| \geq 16 \mathrm{~m}$. Thus $\sum_{\gamma \in \Gamma}\left|a_{2}(\gamma)\right| \geq\left|\sum_{\gamma \in \Gamma} \varepsilon(\gamma) a_{2}(\gamma)\right| \geq 16 \mathrm{~m}$. However $D_{4}$ has precisely 16 Hamiltonian cycles. Thus there is some $\gamma_{0} \in \Gamma$ such that $\left|a_{2}\left(\gamma_{0}\right)\right| \geq m$. Now observe that there is a simple closed $Q$ in $K_{2 n}$ such that $\gamma_{0}$ is obtained from $Q$ by collapsing precisely the edges $\overline{v_{1} u_{2}}, \overline{w_{1} v_{2}}, \overline{z_{1} w_{2}}$, and $\overline{u_{1} z_{2}}$. Thus $Q$ has the same knot type as $\gamma_{0}$ and hence $\left|a_{2}(Q)\right| \geq m$.

We can see as follows that for a given $m \in \mathbb{N}$ there is no $n \in \mathbb{N}$ such that every embedding of $K_{n}$ contains a knot $Q$ with $\left|a_{2}(Q)\right|=m$. First observe that if $Q$ is any knot and $R$ is a right handed trefoil knot, then it follows from equation (1) in the introduction that $a_{2}(Q \# R)=a_{2}(Q)+1$. Let $m \in \mathbb{N}$ and $n \in \mathbb{N}$ be fixed, and let $K_{n}$ be embedded in $\mathbb{R}^{3}$. Consider all simple closed curves $Q_{i}$ which are contained in $K_{n}$, and let $q=\min \left\{a_{2}\left(Q_{i}\right), 0\right\} \leq 0$. We modify the embedding of $K_{n}$ by adding $m-q \geq m$ right handed trefoil knots to every edge within a ball which does not intersect the graph elsewhere. We call this modified embedding $K_{n}^{\prime}$. Now for each simple closed curve $Q_{i}^{\prime}$ in $K_{n}^{\prime}$ there is a corresponding simple closed curve $Q_{i}$ in $K_{n}$. Since every simple closed curve in $K_{n}^{\prime}$ contains at least 3 edges, each $Q_{i}^{\prime}$ is a connected sum which has at least $3(m-q)$ more righthanded trefoil knots as summands than $Q_{i}$ had. Thus for every simple closed curve $Q_{i}^{\prime}$ in $K_{n}^{\prime}$, we have $a_{2}\left(Q_{i}^{\prime}\right) \geq a_{2}\left(Q_{i}\right)+3(m-q) \geq 3 m-2 q \geq 3 m$. Thus $K_{n}^{\prime}$ contains no knot $Q^{\prime}$ such that $\left|a_{2}\left(Q^{\prime}\right)\right|=m$.

\section{References}

[CG] J. Conway, C. McA Gordon, Knots and links in spatial graphs, J. of Graph Theory 7 (1983), 445-453.

[FNP] E. Flapan, R. Naimi, J. Pommersheim, Intrinsically triple linked complete graphs, Topology and its Applications 115 (2001), 239-246.

[Fo] J. Foisy, Intrinsically knotted graphs, J. of Graph Theory 39 (2002), 178-187.

[Ka] L.H. Kauffman, The Conway polynomial, Topology 20 (1981), 101-108.

[Sh] M. Shimabara, Knots in certain spatial graphs, Tokyo J. Math. 11 (1988), 405-413.

[TY] K. Taniyama, A. Yasuhara, Realization of knots and links in a spatial graph, Topology and its Applications 112 (2001), 87-109.

Department of Mathematics, Pomona College

Claremont, CA 91711, U.S.A.

Email: eflapan@pomona.edu

Received: 13 March 2002 